\title{
Prevalence and comorbidity of attention deficit hyperactivity disorder in Spain: study protocol for extending a systematic review with updated meta-analysis of observational studies
}

\author{
Ferrán Catalá-López ${ }^{1,2,3^{*}}$ (D) Manuel Ridao ${ }^{4}$, Amparo Núñez-Beltrán ${ }^{5}$, Ricard Gènova-Maleras ${ }^{6}$, Adolfo Alonso-Arroyo ${ }^{7,8}$,
} Rafael Aleixandre-Benavent ${ }^{8,9}$, Miguel A. Catalá ${ }^{10}$ and Rafael Tabarés-Seisdedos ${ }^{2}$

\begin{abstract}
Background: Attention deficit hyperactivity disorder (ADHD) is a childhood-onset disorder characterized by a persistent pattern of symptoms of developmentally inappropriate and impaired inattention and/or hyperactivity/ impulsivity, with difficulties often continuing into adulthood. ADHD can come with other comorbid conditions. The aim of this study will be to quantify the prevalence and comorbidity of ADHD among children, adolescent, and adult population in Spain.
\end{abstract}

Methods/design: We designed and registered a study protocol for an update and expansion of a systematic review and meta-analysis of pooled prevalence data. We will include cross-sectional observational studies reporting prevalence of ADHD in Spain and conducted in the general population, outpatient, and/or school settings. The primary outcome will be the prevalence of ADHD. Secondary outcomes will be the prevalence of any physical or mental comorbidity in association with ADHD. No limitations will be imposed on publication status, study conduct period, and language of dissemination. Comprehensive literature searches will be conducted in multiple electronic databases, including PubMed/MEDLINE, EMBASE, Scopus, Web of Science, PsycINFO, IME - Spanish Medical Index, and IBECS - Spanish Bibliographic Index of Health Sciences. We will also search Google Scholar, dissertation databases, and conference abstracts. Two team members will independently screen all citations, full-text articles, and abstract data. Potential conflicts will be resolved through discussion. The methodological quality (or risk of bias) of individual studies will be appraised using an appropriate tool. If feasible, we will conduct random effects meta-analysis. Prevalence estimates will be stratified according to gender, age, and geographical location. Additional analyses will be conducted to explore the potential sources of heterogeneity (e.g., methodological quality, sample size, diagnostic criteria).

Discussion: This systematic review and meta-analysis of observational data will provide an updated synthesis of the prevalence and comorbidity of ADHD in Spain. This study will also examine factors that may explain potential variations in prevalence data. The findings of this study will be published in a peer-reviewed journal.

\footnotetext{
* Correspondence: ferran_catala@outlook.com

${ }^{1}$ Department of Health Planning and Economics, National School of Public

Health, Institute of Health Carlos III, Madrid, Spain

${ }^{2}$ Department of Medicine, University of Valencia/INCLIVA Health Research

Institute and CIBERSAM, Valencia, Spain

Full list of author information is available at the end of the article
}

(c) The Author(s). 2019 Open Access This article is distributed under the terms of the Creative Commons Attribution 4.0 International License (http://creativecommons.org/licenses/by/4.0/), which permits unrestricted use, distribution, and reproduction in any medium, provided you give appropriate credit to the original author(s) and the source, provide a link to the Creative Commons license, and indicate if changes were made. The Creative Commons Public Domain Dedication waiver (http://creativecommons.org/publicdomain/zero/1.0/) applies to the data made available in this article, unless otherwise stated. 
(Continued from previous page)

Systematic review registration: PROSPERO CRD42018106082.

Keywords: Attention deficit hyperactivity disorder, Comorbidity, Epidemiological study, Meta-analysis, Prevalence, Spain, Systematic review

\section{Background}

Attention deficit hyperactivity disorder (ADHD) is a childhood-onset disorder characterized by a persistent pattern of symptoms of developmentally inappropriate and impaired inattention and/or hyperactivity/impulsivity, with difficulties often continuing into adulthood [1-3]. ADHD is more common in boys than in girls $[1,4]$ and can often come with other conditions (the so-called comorbidity) [5-7]. Considerable debate exists surrounding the diagnosis [8-13] and treatment [14-17] of ADHD. Claims for the condition being overdiagnosed or underdiagnosed underscore the importance of rigorous assessment $[1,11]$. Recent prevalence estimates suggest that ADHD affects about $3-7 \%$ of young people worldwide [18-23], producing considerable impact on health services and the community $[18,24,25]$. However, prevalence estimates of ADHD within and between countries often vary widely $[19,20,26]$, and reports of increases in prevalence further fuel the controversy [19].

Systematic reviews of descriptive epidemiology are important for characterizing the amount and geographical distribution of health problems. Meta-analysis of epidemiological data (whether at a national, regional or global levels) can be useful to get more precise estimates of disease frequency, monitor trends and changes in disease burden over time, and establish a benchmark pooled prevalence but also to examine whether estimates have increased (or decreased) with publication of different study features. Previous systematic reviews and metaanalyses have traditionally assessed the worldwide prevalence of ADHD based on studies from a broad geographic distribution (e.g., North America, Europe, Asia) [19-22]. Very few meta-analyses exist in the biomedical literature reporting ADHD prevalence estimates at the country level [27-29].

In 2011, members of our review team conducted a meta-analysis of ADHD prevalence in Spain from $14 \mathrm{ob}-$ servational studies and more than 13,000 children and adolescent participants [29]. The main findings were published in 2012, suggesting a first pooled prevalence estimate of $6.8 \%$ (95\% confidence interval: 4.9 to $8.8 \%$ ) at the country level [29]. The 2011 review results drew attention in particular to ADHD among children and adolescents [30,31], but did not include aspects such as comorbidity or adult population. In addition, overall findings were limited by clinical and methodological heterogeneity [29]. In recent years, several (new) epidemiological studies have been conducted in different geographical locations and population groups [32-34]. In addition, methods have advanced quickly; planning data extraction and analyses and understanding of the review process have become more sophisticated [35-41]. Therefore, we consider it timely to update and expand on our previous systematic review and meta-analysis [29] with much more detailed analysis of relevant data.

The objective of this study will be to quantify the prevalence and comorbidity of ADHD among children, adolescent, and adult population in Spain and to examine factors that may explain the variations in prevalence based on the latest evidence from descriptive epidemiology.

\section{Methods}

\section{Protocol}

This study protocol is part of an ongoing evidence synthesis project on the descriptive epidemiology and surveillance of neurodevelopmental disorders [42]. The present protocol has been registered within the PROSPERO database (registration number: CRD42018106082) and is being reported in accordance with the reporting guidance provided in the Preferred Reporting Items for Systematic Reviews and Meta-Analyses Protocols (PRISMA-P) statement [39, 40] (see checklist in Additional file 1).

\section{Information source and literature search}

The primary source of literature will be a structured search of major electronic databases, including PubMed/ MEDLINE, EMBASE, Scopus, Web of Science, and PsycINFO, but also national databases including IME - Indice Médico Español [Spanish Medical Index] and IBECS - Indice Bibliográfico Español en Ciencias de la Salud [Spanish Bibliographic Index of Health Sciences]). The secondary source of potentially relevant material will be a search of the gray or difficult to locate literature, including two dissertation databases (TESEO - Base de datos de Tesis Doctorales [Spanish Data Base of Doctoral Thesis Dissertations] and ProQuest Dissertations and Theses Database), Google Scholar, and conference abstracts from selected national or local symposia on mental health, neurology, and pediatrics. We will perform hand-searching of the reference lists of included studies, relevant reviews, national clinical practice guidelines, or other relevant documents (e.g., official documents and government reports). Content experts and authors who 
are prolific in the field will be contacted. The literature searches will be designed and conducted by the review team which includes two experienced health information specialists. Our main literature search will be peerreviewed by a senior health information specialist using the Peer Review of Electronic Search Strategies (PRESS) checklist [41]. The search will include a broad range of terms and keywords related to ADHD, epidemiological studies, and the geographical area "Spain." For the section of geographic area, the search will be based on a previously validated filter to minimize potential bias regarding the indexing of geographical items [43]. This filter is constructed around three complementary approaches: (1) the term "Spain" and its variants in various languages, (2) terms related mainly to region and province place names, and (3) acronyms for regional health services. A draft search strategy for PubMed/MEDLINE is provided in Additional file 2.

\section{Eligibility criteria}

Studies will be selected according to the following criteria: participants, condition or outcome(s) of interest, study design, and context.

- Participants (population): We will include studies involving children, adolescents, and adult population (regardless of age or sex).

- Condition or outcome(s) of interest: The primary outcome will be the prevalence of ADHD indicating the number of people that have the disorder divided by the population number at a given point in time. This is often presented as a (prevalence) proportion. We will use author-reported definitions (according to accepted diagnostic criteria, such as the Diagnostic and Statistical Manual of Mental Disorders [DSM] or the International Classification of Diseases [ICD] criteria: ICD-9: 314.00, 314.01; ICD-10: F90). Secondary outcomes will be the prevalence of any comorbidity, indicating the existence of any distinct additional (physical or mental) condition in association with ADHD (e.g., according to main DSM-IV, DSM-V, ICD-9, or ICD-10 categories of diagnoses).

- Study design and context: Eligible studies will be observational studies (cross-sectional or health surveys) reporting prevalence data using validated or non-validated tools and conducted in a wide range of people in the Spanish general population, outpatient (including data from administrative databases and registries), and/or school settings. Cross-sectional studies will be the most appropriate study design to determine the prevalence of ADHD. Cross-sectional health surveys are typically used to estimate the point prevalence of common conditions of long duration. We will exclude studies in hospital/inpatient clinical settings because they are likely to be highly selected resulting in inaccurate estimations of the "true prevalence" of the disorder.

No limitations will be imposed on language, publication status (unpublished studies will be eligible for inclusion), and study conduct period.

\section{Screening and selection procedure}

All articles identified from the literature search will be screened by two team members independently. First, titles and abstracts of articles returned from initial searches will be screened based on the eligibility criteria outlined above. Second, full texts will be examined in detail and screened for eligibility. Third, references of all considered articles will be hand-searched to identify any relevant report missed in the search strategy. Any disagreements will be resolved by discussion to meet a consensus, if necessary. A flow chart showing details of studies included and excluded at each stage of the study selection process will be provided [44].

\section{Data collection}

A data extraction form will be designed and used to extract equivalent information from each study report. Information of interest will include the following:

- Study characteristics: study design, year of publication, journal, year (or period) of study conduct, sample size, setting (community, school or outpatient), geographical location of study conduct: North (Galicia, Asturias, Cantabria, Aragon, Basque Country, Navarre, La Rioja), Mediterranean (Balearics, Catalonia, Valencia), Centre (Castile-La Mancha, Castile-León, Madrid, Extremadura), and South-East (Andalusia, Murcia and Canary Islands); and other fields to capture data relevant to the assessment of study methodological quality (see risk of bias assessment subsection).

- Participant characteristics: population sampled, age (e.g., mean with standard deviation, range) and gender (e.g., percentage of female participants).

- Outcome results: definitions and measures used to make diagnosis (e.g., symptom only checklists, reports of diagnosis, interviews and examination) and whether the diagnosis met the full DSM/ICD criteria for each edition (e.g., DSM-III, DSM-IV, DSM-V, ICD-9, ICD-10, ICD-11, other), informants or people reporting symptoms for a diagnostic evaluation (e.g., child, clinician, parent, teacher and/ or a combination rule), prevalence estimates (e.g., number of subjects with the disorder, proportion and $95 \%$ confidence interval), and any prevalence estimates stratified by age, gender, severity (impairment criterion for DSM-IV), or location. The most 
conservative diagnosis will be used in those studies reporting more than one prevalence estimate. If outcome results (e.g., proportion and 95\% confidence interval) are not directly provided and it is feasible, we will calculate them from the number of cases and sample size provided in each single study.

Data extraction forms will be piloted initially on a small number of included studies. Subsequently, each of the included studies will be abstracted by two team members, independently, and potential conflicts will be resolved through discussion. Authors of primary publications (e.g., corresponding authors) will be contacted by email for data clarifications or missing outcome data, as necessary. First, authors will be sent an email requesting their missing outcome data or data clarifications. Second, we will send three email reminders at 2-, 6-, and 10-week intervals after the initial email. In cases where the identified studies do not report authors' email addresses or include non-working email addresses, we will search authors' publications, PubMed, and profiles that are publicly available (e.g., ORCID, ResearchGate, and Google Scholar), to find contact information.

\section{Risk of bias in individual studies}

The risk of bias in individual studies will be evaluated using a methodological quality critical appraisal checklist proposed by the Joanna Briggs Institute (JBI) systematic review methods manual $[37,38]$. This tool for observational studies reporting prevalence data considers the following: sample representativeness, recruitment appropriateness, sample size, description of subjects and setting, coverage of data analysis, ascertainment and measurement of the condition, thoroughness of reporting statistical analysis, and identification and accountability of potential confounding factors/subgroups (see Additional file 3). Stars or points will be awarded for each quality item, and the highest quality studies will be awarded up to ten stars. Studies will be judged to be at low risk of bias ( $\geq 7$ points), moderate risk of bias (4-6 points), or high risk of bias ( $<4$ points) [42]. The risk of bias for each individual study will be independently assessed by two reviewers. Discrepant scores will be resolved by discussion and consensus. We will provide a narrative summary of the risk of bias of the included studies, which will be supported by a table showing the results of the critical appraisal.

\section{Methods for evidence synthesis}

The data from each paper (e.g., study characteristics, context, participants, outcomes and findings) will be used to build evidence tables of an overall description of included studies. Crude prevalence estimates (number of cases/sample size) will be presented along with $95 \%$ confidence intervals. If feasible and appropriate, prevalence data points from primary observational studies will be used to perform random effects meta-analyses. Since heterogeneity is expected a priori, we will estimate the pooled prevalence and its 95\% confidence interval using the random effects model with logit transformation and back transformation [42]. The random effects model assumes the study prevalence estimates follow a normal distribution, considering both within-study and between-study variation. Forest plots will be used to visualize pooled estimates and the extent of heterogeneity among studies.

We will quantify statistical heterogeneity by estimating the variance between studies using $I^{2}$ statistic [45]. The $I^{2}$ statistic is the proportion of variation in prevalence estimates that is due to genuine variation in prevalence rather than sampling (random) error. $I^{2}$ statistic ranges between 0 and $100 \%$ (with values of $0-25 \%$ and $75-$ $100 \%$ taken to indicate low and considerable heterogeneity, respectively). We will also report $\mathrm{Tau}^{2}$ [46] and Cochran $\mathrm{Q}$ test [47] with a $P$ value of $<0.05$ considered statistically significant (heterogeneity).

\section{Additional analyses}

If sufficient studies are identified and data points are available, potential sources of heterogeneity will be investigated further by subgroup or meta-regression analyses [48] according to baseline characteristics and methodological covariates $[1-4,19,29,42]$. We plan to conduct analyses by gender (male vs female), age (e.g., children vs adolescent vs adult, mid-point of age range as continuous variable), geographical location (e.g., North, Mediterranean, Centre, and South-East), setting (e.g., community/school vs outpatient), sample size (e.g., <500, 500-1500, or > 1500 participants), decade of publication (e.g., 1990, 2000, or 2010), study quality (e.g., low/moderate vs high risk of bias), diagnostic system (e.g., DSM vs ICD criteria), and most recent diagnostic criteria (e.g., "DSM-IV/V or ICD-10/11" vs "Not DSM-IV/V or ICD-10/11"). In addition, we will explore prevalence trends with gender variations (in terms of the female-to-male prevalence ratios) and over time (with the year of publication as the explanatory variable) using random effects meta-regression models [29].

\section{Meta-bias}

Small study effects (or "publication bias" across studies) will be assessed by inspection of the funnel plots for asymmetry and with Egger's test [49] and Begg's test [50], with the results considered to indicate potential small study effects when $P$ values are $<0.10$. 


\section{Software considerations}

All analyses will be conducted in Stata version 15 (StataCorp LP, College Station, Texas, USA) [51, 52].

\section{Patient and public involvement}

We will evaluate whether the epidemiological studies included in the systematic review had any patient and public involvement $[53,54]$.

No patients and/or public were involved in setting the research question for this study nor were they involved in developing plans for design (or implementation) of this protocol. No patients and/or public will be asked to advise on interpretation or writing up of results.

\section{Ethics, dissemination, and research integrity}

No ethical approval is required for the performance of this study. The proposed systematic review and meta-analysis will be reported in accordance with the reporting guidance provided in the Preferred Reporting Items for Systematic Reviews and Meta-analyses (PRISMA) statement [44] and the Meta-analysis Of Observational Studies in Epidemiology (MOOSE) reporting guideline [55]. Any amendments made to this protocol when conducting the study will be outlined and reported in the final manuscript. Results will be disseminated through conference presentations and publication in a peer-reviewed journal. All data underlying the findings reported in the final manuscript will be deposited in a cross-disciplinary public repository-such as the Open Science Framework (https://osf.io/) or Zenodo (https:// zenodo.org/).

\section{Discussion}

Up-to-date epidemiological evidence about levels and trends in country-specific morbidity (such as prevalence) is essential input into national and subnational health policy and planning debates. In fact, the availability of national prevalence estimates of ADHD provides opportunities to undertake further systematic assessments of the burden of disease [18, 23, 25].

In this paper, we have presented a study protocol for extending a systematic review with updated metaanalysis of the prevalence and comorbidity of ADHD in Spain. This protocol updates and expands methods for a new systematic review that will supersede previous meta-analyses of observational studies on this topic [29]. The improved approaches to the methods, analysis, and refinements in epidemiological data (revisions and updates, exploration of the extent of bias, heterogeneity, and potential variations in prevalence data), as well as the widening of scope by age (e.g., children, adolescents and adults), causes of comorbidity (e.g., physical or mental), and time considering new data (e.g., studies from 1980 to 2020) are all relevant to this study.
A key challenge is that based on knowledge from previous systematic reviews on mental health [19-24, 56, 57], we anticipate identifying epidemiological studies with different features, populations, and contexts and with a variable quality of reporting methods and results. This study will identify knowledge gaps to be filled by new research. On this regard, implications for future epidemiological studies will be discussed in the final manuscript.

\section{Additional files}

Additional file 1: PRISMA-P Checklist. (DOCX $33 \mathrm{~kb}$ )

Additional file 2: Key terms for PubMed/MEDLINE search. (DOCX 29 kb)

Additional file 3: Methodological Quality Checklist for Prevalence data. (DOCX $29 \mathrm{~kb}$ )

\section{Abbreviations}

ADHD: Attention deficit hyperactivity disorder; DSM: Diagnostic and Statistical Manual of Mental Disorders; ICD: International Classification of Diseases; MOOSE: Meta-analysis Of Observational Studies in Epidemiology; PRISMA: Preferred Reporting Items for Systematic Reviews and Meta-Analyses; PRISMA-P: Preferred Reporting Items for Systematic Reviews and Meta-Analyses extension for Protocols

\section{Funding}

FC-L and RT-S are funded by CIBERSAM/Institute of Health Carlos III. MR is partially funded by the Spanish Health Services Research on Chronic Patients Network (REDISSEC)/Institute of Health Carlos III. The funders were not involved in the design of the protocol or decision to submit the protocol for publication, nor will they be involved in any aspect of the conduct of the study.

\section{Availability of data and materials \\ Not applicable.}

\section{Authors' contributions}

The study protocol was conceived by FC-L, with critical input from MR, AN-B, RG-M, AA-A, RA-B, MAC, and RT-S. FC-L registered the protocol with the PROSPERO database and wrote the first draft of the protocol. MR, MAC, and AN-B provided input into the design and edited the draft protocol. All authors commented on the paper for important intellectual content. All authors read and approved the final paper. FC-L accepts full responsibility for the finished paper and controlled the decision to publish. FC-L is the principal investigator and guarantor.

\section{Authors' information}

FC-L is a PhD (Public Health) and MPH (Quantitative Methods). MR is a PhD (Medicine) and MSc (Health Economics). AN-B is PsyD (Clinical Psychology) and MSc (Neurosciences). RG-M is a MSc (Demography). AA-A is a PhD (Information and Documentation) and MA. RA-B is a MD and PhD (Medical Documentation). MAC is a MD (Child Psychiatry) and PhD (Medical Documentation). RT-S is a MD (Psychiatry) and PhD.

Ethics approval and consent to participate Not applicable.

\section{Consent for publication}

Not applicable.

\section{Competing interests}

The authors declare that they have no competing interests.

\section{Publisher's Note}

Springer Nature remains neutral with regard to jurisdictional claims in published maps and institutional affiliations. 


\section{Author details}

'Department of Health Planning and Economics, National School of Public Health, Institute of Health Carlos III, Madrid, Spain. ${ }^{2}$ Department of Medicine, University of Valencia/INCLIVA Health Research Institute and CIBERSAM, Valencia, Spain. ${ }^{3}$ Fundación Instituto de Investigación en Servicios de Salud, Valencia, Spain. ${ }^{4}$ Instituto Aragonés de Ciencias de la Salud (IACS), Red de Investigación en Servicios de Salud en Enfermedades Crónicas (REDISSEC) Zaragoza, Spain. ${ }^{5}$ Independent Clinical Psychologist and Researcher, Madrid, Spain. ${ }^{6}$ Directorate General for Public Health, Madrid Regional Health Council, Madrid, Spain. ${ }^{7}$ Department of History of Science and Documentation, University of Valencia, Valencia, Spain. ${ }^{8}$ Information and Social and Health Research Unit (UISYS), University of Valencia and Spanish National Research Council (CSIC), Valencia, Spain. ${ }^{9}$ Institute for Innovation and Knowledge Management (INGENIO)/Spanish National Research Council (CSIC) and Polytechnic University of Valencia (UPV), Valencia, Spain. ${ }^{10}$ Faculty of Medicine, University of Valencia, Valencia, Spain.

Received: 29 August 2018 Accepted: 28 January 2019

Published online: 11 February 2019

\section{References}

1. Thapar A, Cooper M. Attention deficit hyperactivity disorder. Lancet. 2016 ; 387(10024):1240-50. https://doi.org/10.1016/S0140-6736(15)00238-X.

2. Verkuiil N, Perkins M, Fazel M. Childhood attention-deficit/hyperactivity disorder. BMJ. 2015;350:h2168. https://doi.org/10.1136/bmj.h2168.

3. Feldman HM, Reiff MI. Clinical practice. Attention deficit-hyperactivity disorder in children and adolescents. N Engl J Med. 2014;370(9):838-46. https://doi.org/10.1056/NEJMcp1307215.

4. Biederman J, Kwon A, Aleardi M, Chouinard VA, Marino T, Cole H, et al. Absence of gender effects on attention deficit hyperactivity disorder: findings in nonreferred subjects. Am J Psychiatry. 2005;162(6):1083-9. https://doi.org/10.1176/appi.ajp.162.6.1083.

5. Muskens JB, Velders FP, Staal WG. Medical comorbidities in children and adolescents with autism spectrum disorders and attention deficit hyperactivity disorders: a systematic review. Eur Child Adolesc Psychiatry. 2017. 26(9):1093-103. https://doi.org/10.1007/s00787-017-1020-0.

6. Tung I, Li JJ, Meza JI, Jezior KL, Kianmahd JS, Hentschel PG, O'Neil PM, Lee SS. Patterns of comorbidity among girls with ADHD: a meta-analysis. Pediatrics. 2016;138(4):e20160430.

7. Catalá-López F, Alonso-Arroyo A, Page MJ, Hutton B, Tabarés-Seisdedos R, Aleixandre-Benavent R. Mapping of global scientific research in comorbidity and multimorbidity: a cross-sectional analysis. PLoS One. 2018;13(1):e0189091. https://doi.org/10.1371/journal.pone.0189091.

8. Brodersen J, Schwartz LM, Heneghan C, O'Sullivan JW, Aronson JK, Woloshin S. Overdiagnosis: what it is and what it isn't. BMJ Evid Based Med. 2018; 23(1):1-3. https://doi.org/10.1136/ebmed-2017-110886

9. Bowers S. Why are we failing young patients with ADHD? BMJ. 2014;349: g6082. https://doi.org/10.1136/bmj.g6082.

10. Thomas R, Mitchell GK, Batstra L. Attention-deficit/hyperactivity disorder: are we helping or harming? BMJ. 2013;347:f6172. https://doi.org/10.1136/bmj. f6172.

11. Taylor E. Attention deficit hyperactivity disorder: overdiagnosed or diagnoses missed? Arch Dis Child. 2017;102(4):376-9. https://doi.org/10.1136/archdischild2016-310487.

12. Coon ER, Quinonez RA, Moyer VA, Schroeder AR. Overdiagnosis: how our compulsion for diagnosis may be harming children. Pediatrics. 2014;134(5): 1013-23. https://doi.org/10.1542/peds.2014-1778.

13. Morrow RL, Garland EJ, Wright JM, Maclure M, Taylor S, Dormuth CR. Influence of relative age on diagnosis and treatment of attentiondeficit/hyperactivity disorder in children. CMAJ. 2012;184(7):755-62 https://doi.org/10.1503/cmaj.111619.

14. Hoekstra PJ, Buitelaar JK. Is the evidence base of methylphenidate for children and adolescents with attention-deficit/hyperactivity disorder flawed? Eur Child Adolesc Psychiatry. 2016 Apr;25(4):339-40. https://doi. org/10.1007/s00787-016-0845-2.

15. Storebø OJ, Simonsen E, Gluud C. The evidence base of methylphenidate for children and adolescents with attention-deficit hyperactivity disorder is in fact flawed. Eur Child Adolesc Psychiatry. 2016;25(9):1037-8. https://doi. org/10.1007/s00787-016-0855-0

16. The Lancet. The realities and needs of children living with ADHD. Lancet. 2014;384(9938):104. https://doi.org/10.1016/S0140-6736(14)61146-6.
17. McGough JJ. Treatment controversies in adult ADHD. Am J Psychiatry. 2016; 173(10):960-6. https://doi.org/10.1176/appi.ajp.2016.15091207.

18. GBD 2016 Disease and Injury Incidence and Prevalence Collaborators. Global, regional, and national incidence, prevalence, and years lived with disability for 328 diseases and injuries for 195 countries, 1990-2016: a systematic analysis for the Global Burden of Disease Study 2016. Lancet. 2017;390(10100):1211-59. https://doi.org/10.1016/S0140-6736(17)32154-2.

19. Thomas R, Sanders S, Doust J, Beller E, Glasziou P. Prevalence of attentiondeficit/hyperactivity disorder: a systematic review and meta-analysis. Pediatrics. 2015;135(4):e994-1001. https://doi.org/10.1542/peds.2014-3482.

20. Polanczyk GV, Willcutt EG, Salum GA, Kieling C, Rohde LA. ADHD prevalence estimates across three decades: an updated systematic review and metaregression analysis. Int J Epidemiol. 2014;43(2):434-42. https://doi.org/10. 1093/ije/dyt261

21. Willcutt EG. The prevalence of DSM-IV attention-deficit/hyperactivity disorder: a meta-analytic review. Neurotherapeutics. 2012;9(3):490-9. https://doi.org/10. 1007/s13311-012-0135-8.

22. Polanczyk G, de Lima MS, Horta BL, Biederman J, Rohde LA. The worldwide prevalence of ADHD: a systematic review and metaregression analysis. Am J Psychiatry. 2007;164(6):942-8. https://doi.org/10.1176/ajp.2007.164.6.942.

23. Erskine HE, Ferrari AJ, Nelson P, Polanczyk GV, Flaxman AD, Vos T, et al. Epidemiological modelling of attention-deficit/hyperactivity disorder and conduct disorder for the global burden of disease study 2010. J Child Psychol Psychiatry. 2013;54(12):1263-74. https://doi.org/10.1111/jcpp.12144.

24. Erskine HE, Ferrari AJ, Polanczyk GV, Moffitt TE, Murray CJ, Vos T, et al. The global burden of conduct disorder and attention-deficit/hyperactivity disorder in 2010. J Child Psychol Psychiatry. 2014;55(4):328-36. https://doi. org/10.1111/jcpp.12186.

25. Global Burden of Disease Child and Adolescent Health Collaboration, Kassebaum N, Kyu HH, Zoeckler L, Olsen HE, Thomas K, et al. Child and adolescent health from 1990 to 2015: findings from the Global Burden Diseases, Injuries, and Risk Factors 2015 Study. JAMA Pediatr. 2017:171(6): 573-92. https://doi.org/10.1001/jamapediatrics.2017.0250

26. Erskine HE, Baxter AJ, Patton G, Moffitt TE, Patel V, Whiteford HA, et al. The global coverage of prevalence data for mental disorders in children and adolescents. Epidemiol Psychiatr Sci. 2017;26(4):395-402. https://doi.org/10. 1017/S2045796015001158

27. Wang T, Liu K, Li Z, Xu Y, Liu Y, Shi W, Chen L. Prevalence of attention deficit/hyperactivity disorder among children and adolescents in China: a systematic review and meta-analysis. BMC Psychiatry. 2017;17(1):32. https:// doi.org/10.1186/s12888-016-1187-9.

28. Alhraiwil NJ, Ali A, Househ MS, Al-Shehri AM, El-Metwally AA. Systematic review of the epidemiology of attention deficit hyperactivity disorder in Arab countries. Neurosciences. 2015;20(2):137-44. https://doi.org/10.17712/ nsj.2015.2.20140678

29. Catalá-López F, Peiró S, Ridao M, Sanfélix-Gimeno G, Gènova-Maleras R, Catalá MA. Prevalence of attention deficit hyperactivity disorder among children and adolescents in Spain: a systematic review and meta-analysis of epidemiological studies. BMC Psychiatry. 2012;12:168. https://doi.org/10. 1186/1471-244X-12-168.

30. Program of Activities for the Early Detection of Health Problems between 0 and 14 years-old: Effectiveness, safety and economic evaluation of the existing alternatives for the early detection of Attention Deficit Hyperactivity Disorder (ADHD). Reports of Health Technology Assessment. Health Sciences Institute in Aragón (IACS). Madrid: Ministry of Health, Social Services and Equality; 2016. http://www.iacs.es/wp-content/uploads/2017/ 05/758-IACS-TDAH.pdf. Accessed 1 Feb 2019.

31. $A B C$ [News]. El 6,8\% de los niños españoles padecería trastorno por déficit de atención e hiperactividad. December 12, 2013. http://www.abc.es/salud/ noticias/20131111/abci-ninos-espanoles-padeceria-trastorno-201311291110. html. Accessed 22 June 2018.

32. Carballal Mariño M, Gago Ageitos A, Ares Alvarez J, Del Rio Garma M, García Cendón C, Goicoechea Castaño A, et al. Prevalence of neurodevelopmental, behavioural and learning disorders in Pediatric Primary Care. An Pediatr (Barc). 2017 doi: https://doi.org/10.1016/j.anpedi.2017.10.007.

33. Canals J, Morales-Hidalgo $P$, Jané MC, Domènech $E$. ADHD prevalence in Spanish preschoolers: comorbidity, socio-demographic factors, and functional consequences. J Atten Disord. 2018;22(2):143-53. https://doi.org/10.1177/ 1087054716638511.

34. Cerrillo-Urbina AJ, García-Hermoso A, Martínez-Vizcaíno V, Pardo-Guijarro MJ Ruiz-Hermosa A, Sánchez-López M. Prevalence of probable attention-deficit/ 
hyperactivity disorder symptoms: result from a Spanish sample of children BMC Pediatr. 2018;18(1):111. https://doi.org/10.1186/s12887-018-1083-1.

35. An integrative metaregression framework for descriptive epidemiology. Flaxman AD, Vos T, Murray CJL (Editors). Seattle: University of Washington Press; 2015. http:/www.washington.edu/uwpress/search/books/FLAINT.html.

36. Barendregt JJ, Doi SA, Lee YY, Norman RE, Vos T. Meta-analysis of prevalence. J Epidemiol Community Health. 2013;67(11):974-8. https://doi.org/10.1136/jech2013-203104.

37. Munn Z, Moola S, Lisy K, Riitano D, Tufanaru C. Chapter 5: Systematic reviews of prevalence and incidence. In: Aromataris E, Munn Z (Editors). Joanna Briggs Institute Reviewer's Manual. The Joanna Briggs Institute; 2017. https://wiki. joannabriggs.org/. Accessed 22 June 2018.

38. Munn Z, Moola S, Lisy K, Riitano D, Tufanaru C. Methodological guidance for systematic reviews of observational epidemiological studies reporting prevalence and cumulative incidence data. Int J Evid Based Healthc. 2015; 13(3):147-53. https://doi.org/10.1097/XEB.0000000000000054.

39. Moher D, Shamseer L, Clarke M, Ghersi D, Liberati A, Petticrew M, et al. Preferred reporting items for systematic review and meta-analysis protocols (PRISMA-P) 2015 statement. Syst Rev. 2015;4:1. https://doi.org/10.1186/20464053-4-1.

40. Shamseer L, Moher D, Clarke M, Ghersi D, Liberati A, Petticrew M, et al. Preferred reporting items for systematic review and meta-analysis protocols (PRISMA-P) 2015: elaboration and explanation. BMJ. 2015;350:g7647. https:// doi.org/10.1136/bmj.g7647.

41. McGowan J, Sampson M, Salzwedel DM, Cogo E, Foerster V, Lefebvre C. PRESS peer review of electronic search strategies: 2015 guideline statement. J Clin Epidemiol. 2016;75:40-6. https://doi.org/10.1016/j.jclinepi.2016.01.021.

42. Catalá-López F, Ridao M, Hurtado I, Núñez-Beltrán A, Gènova-Maleras R, AlonsoArroyo A, et al. Prevalence and comorbidity of autism spectrum disorder in Spain: study protocol for a systematic review and meta-analysis of observational studies. PROSPERO 2018 CRD42018090372. http:/www.crd.york.ac.uk/PROSPERO/display_ record.php?|D=CRD42018090372. Accessed 1 Feb 2019.

43. Valderas JM, Mendivil J, Parada A, Losada-Yáñez M, Alonso J. Development of a geographic filter for PubMed to identify studies performed in Spain. Rev Esp Cardiol. 2006;59(12):1244-51. https://doi.org/10.1016/S18855857(07)60080-2.

44. Moher D, Liberati A, Tetzlaff J, Altman DG, PRISMA Group. Preferred reporting items for systematic reviews and meta-analyses: the PRISMA statement. PLoS Med. 2009;6(7):e1000097. https://doi.org/10.1371/journal. pmed.1000097.

45. Higgins JP, Thompson SG, Deeks JJ, Altman DG. Measuring inconsistency in meta-analyses. BMJ. 2003;327(7414):557-60. https://doi.org/10.1136/bmj.327. 7414.557.

46. Deeks JJ, Higgins JPT, Altman DG. Chapter 9: Analysing data and undertaking meta-analyses. In: JPT H, Green S, editors. Cochrane handbook for systematic reviews of interventions. Chichester (UK): Wiley; 2008.

47. Cochran WG. The combination of estimates from different experiments. Biometrics. 1954;10:101-29. https://doi.org/10.2307/3001666.

48. Harbord RM, Higgins JPT. Meta-regression in Stata. Stata J. 2008;8(4):493-519.

49. Egger M, Davey Smith G, Schneider M, Minder C. Bias in meta-analysis detected by a simple, graphical test. BMJ. 1997;315(7109):629-34. https:// doi.org/10.1136/bmj.315.7109.629.

50. Begg CB, Mazumdar M. Operating characteristics of a rank correlation test for publication bias. Biometrics. 1994;50(4):1088-101. https://doi.org/10. 2307/2533446

51. StataCorp: Stata Statistical Software: Release 15. College Station, TX: StataCorp LP; 2017

52. Palmer TM, Sterne JAC, Newton HJ, Cox NJ (Editors). Meta-analysis in Stata: an updated collection from the Stata journal. Second ed. College Station, TX: StataCorp LP; 2016.

53. Staniszewska S, Brett J, Simera I, Seers K, Mockford C, Goodlad S, et al GRIPP2 reporting checklists: tools to improve reporting of patient and public involvement in research. BMJ. 2017;358:j3453. https://doi.org/10. 1136/bmj.j3453.

54. Brett J, Staniszewska S, Simera I, Seers K, Mockford C, Goodlad S, et al. Reaching consensus on reporting patient and public involvement (PPI) in research: methods and lessons learned from the development of reporting guidelines. BMJ Open. 2017;7(10):e016948. https://doi.org/10.1136/bmjopen2017-016948.

55. Stroup DF, Berlin JA, Morton SC, Olkin I, Williamson GD, Rennie D, et al. Meta-analysis of observational studies in epidemiology: a proposal for reporting. Meta-analysis of observational studies in epidemiology (MOOSE) group. JAMA. 2000;283(15):2008-12. https://doi.org/10.1001/jama.283.15.2008.

56. Catalá-López F, Hutton B, Núñez-Beltrán A, Page MJ, Ridao M, Macías SaintGerons $\mathrm{D}$, et al. The pharmacological and non-pharmacological treatment of attention deficit hyperactivity disorder in children and adolescents: a systematic review with network meta-analyses of randomised trials. PLoS One. 2017;12(7):e0180355. https://doi.org/10.1371/journal.pone.0180355.

57. Catalá-López F, Hutton B, Page MJ, Ridao M, Driver JA, Alonso-Arroyo A, et al. Risk of mortality among children, adolescents, and adults with autism spectrum disorder or attention deficit hyperactivity disorder and their firstdegree relatives: a protocol for a systematic review and meta-analysis of observational studies. Syst Rev. 2017;6(1):189. https://doi.org/10.1186/ s13643-017-0581-9.

\section{Ready to submit your research? Choose BMC and benefit from:}

- fast, convenient online submission

- thorough peer review by experienced researchers in your field

- rapid publication on acceptance

- support for research data, including large and complex data types

- gold Open Access which fosters wider collaboration and increased citations

- maximum visibility for your research: over $100 \mathrm{M}$ website views per year

At $\mathrm{BMC}$, research is always in progress.

Learn more biomedcentral.com/submissions 